\title{
MediBot: An ontology based chatbot for portuguese speakers drug's users
}

\author{
Caio Viktor S. Avila ${ }^{1}$, Anderson B. Calixto ${ }^{1}$, Tulio Vidal Rolim ${ }^{1}$, Wellington Franco ${ }^{2}$, Amanda D. P. \\ Venceslau $^{2}$, Vânia M. P. Vidal ${ }^{1}$, Valéria M. Pequeno ${ }^{3}$, and Francildo Felix De Moura ${ }^{1}$ \\ ${ }^{1}$ Department of Computing, Federal University of Ceará, Campus do Pici, Fortaleza-CE, Brazil \\ ${ }^{2}$ Federal University of Ceará, Campus de Crateús, Crateús-CE, Brazil \\ ${ }^{3}$ TechLab, Departamento de Ciências e Tecnologias, Universidade Autónoma de Lisboa Luís de Camões, Portugal \\ caioviktor@alu.ufc.br, \{andersonbr,vvidal\}@lia.ufc.br, \{tulio.xcrtf,francildofelix\}@gmail.com,wellington@crateus.ufc.br, \\ amanda.pires@ufc.br,vpequeno@autonoma.pt
}

Keywords: Chatbot, Data Integration, Semantic Web, Medical Informatics, Drugs .

\begin{abstract}
Brazil is one of the countries with the highest level of drug consumption in the world. By 2012 about $66 \%$ claimed to practice self-medication. Such activity can lead to a wide range of risks, including death from drug intoxication. Studies indicate that a lack of knowledge about drugs and their dangers is one of the main aggravating factors in this scenario. This work aims to universalize access to information about medications and their risks for different user profiles, especially Brazilian and lay users. In this paper, we presented the construction process of a Linked Data Mashup (LDM) integrating the datasets: consumer drug prices, government drug prices and drug's risks in pregnant from ANVISA and SIDER from BIO2RDF. In addition, this work presents MediBot, an ontology-based chatbot capable of responding to requests in natural language in Portuguese through the instant messenger Telegram, smoothing the process to query the data. MediBot acts like a native language query interface on an LDM that works as an abstraction layer that provides an integrated view of multiple heterogeneous data sources.
\end{abstract}

\section{Introduction}

Brazil is the fifth country with the highest consumption of drugs in the world, being the first in Latin America (Sousa et al., 2008). In the nation, drugs occupy the first position among the agents causing intoxication, in front of toxic drugs such as pesticides, illicit drugs, rodenticides, insecticides and foodstuffs improper for consumption (Corrêa et al., 2013). In 2012, according to Pinto et al. (2012), 66\% of the population said he had practiced self-medication in his lifetime. It is understood as self-medication the use of drugs without any intervention by a physician, or other qualified professional, neither in diagnosis, nor in prescription, nor the follow-up of treatment. Only in 2016, there were approximately 56,937 registered cases of human intoxication, of which, 226 evolved to death, 20,527 being generated due to the use of drugs (SINITOX, 2016).

The pharmaceutical professional is of great importance in combating the risks resulting from selfmedication. However, in many cases access to a pharmacist perhaps impracticable, either because of the low number of professionals relative to the population size or because of the distance in more remote areas. Also, there is a need for public awareness campaigns about the risks of adverse effects of certain drugs.

Authors claim that actions such as improving the quality of prescriptions and preventing inappropriate self-medication could reduce the occurrence of adverse reactions (Queneau et al., 2007). Another problem is that the health terminologies used are represented using two perspectives: the end-user and the health professional, making it difficult to understand medical terminologies and their classifications. Therefore, a common vocabulary is necessary, making possible the understanding of medical terminologies and their classifications with a single meaning.

In the web, there is a wide variety of data about drugs, such data originating from either government organizations, such as regulatory agencies and public data portals, makers, on-line drugstores and electronic bulletin. However, the vast majority of these data are in the proprietary format, such as spreadsheets, relational database backups or are available only through web pages. Also, such data are isolated in data silos, 
with no direct connection between resources. Moreover, each dataset perhaps represented in different vocabularies, where the same concept in the real world is represented with varying terms through different datasets.

An example of information that can not be retrieved directly in the current state of data is given in next: "What are the risks of the drug XX?". This simple query requires information from a different set of sources. The retrieval of such information has some challenges, such as heterogeneity of the format of the sources, where some that are founded in $X L S, C S V$, $R D F$ formats or web pages which requires different query techniques. Besides, there's the need to perform the information junction manually, since such databases usually aren't integrated. Moreover, it is necessary for the user to have technical knowledge about the area, as there may be a need to resolve vocabulary inconsistencies. These problems can be solved by using Semantic Web technologies, such as ontologies that allow the data's representation in the same target vocabulary.

Semantic Web (Shadbolt et al., 2006) and Linked Data (Bizer et al., 2011) technologies are used to handle such limitations. This technology allows a semantic integration between resources in different sources, representing the data in a unified vocabulary through the use of ontologies. The ontology provides a uniform vocabulary for data access, acting as an abstraction layer for data access. Besides, it allows its publication openly in a non-proprietary format that allows retrieval of information by both computerized agents and human users via $S P A R Q L$ queries endpoints.

The main aim of this work is to facilitate access to information about drugs and their risks easily and directly to users. For this purpose we performed the integration of drug data with a focus on Brazilian data, finally producing a Linked Data Mashup (Hoang et al., 2014), and as an application an ontology-based chatbot, called MediBot is presented to the Telegram instant messenger to answer questions in natural language about the integrated data, providing a more intuitive user interface. The main contributions are:

- A data's integration of different data sources about drugs. We add the information on drug risks contained in the SIDER dataset to the products sold only in Brazil;

- A mashup model to integrate drug bases;

- A Natural Language Interface (NLI) for end-user through the use of a ChatBot.

The rest of this paper is organized as follows: Section 2 presents the related works. Section 3 describes the data integration process. Section 4 introduces
Medibot, a chatbot application for queries about the integrated data. In section 5 we present the evaluation of MediBot. Finally, in section 6, are discussed the conclusions and future work.

\section{Related works}

In (Jovanovik, 2017), it showed to perform the integration and publication of data about drugs of twenty and three countries. However, Brazil is not found between these. Also, the vocabulary defined for the integration does not fit the databases selected in this work.

(Natsiavas et al., 2017) present a model for use of several databases integrated through Linked Data, in particular, datasets of the project $B I O 2 R D F$ for the mining of signs of adverse reactions of drugs, showing the potential of linked databases in the drug domain. However, the model was designed to be used as part of a data mining platform, so it does not address how users will access data. In addition to this work, others have the same goal as (Nováček et al., 2017) and (Natsiavas et al., 2015).

(Vega-Gorgojo et al., 2016) presents PepeSearch, a system that facilitates searching between different sources Linked Data in the field of drugs and health, such as Drug Bank(DB) and Sider. The system provides a faceted query interface that allows the user to search on multiple data sources. However, the system is best suited for use by specialists and researchers, besides it has a powerful yet complex query interface. Moreover, the system does not have data on drug risks in pregnancy (DRP), despite having the SIDER side-effects data. Lastly, "MedChatBot" is a chatbot for medical students based on the open source AIML UMLS to generate responses to queries through knowledge extraction. Table 1 presents an analysis of the aspects present in this work and the others related (Kazi et al., 2012).

Table 1: Comparison of Related Work.

\begin{tabular}{|c|c|c|c|c|}
\hline & DB & Sider & DRP & NLI \\
\hline (Jovanovik, 2017) & $\mathrm{X}$ & $\mathrm{X}$ & - & - \\
\hline (Natsiavas et al., 2017) & $\mathrm{X}$ & $\mathrm{X}$ & - & - \\
\hline (Nováček et al., 2017) & $\mathrm{X}$ & $\mathrm{X}$ & - & - \\
\hline (Natsiavas et al., 2015) & $\mathrm{X}$ & $\mathrm{X}$ & - & - \\
\hline (Vega-Gorgojo et al., 2016) & $\mathrm{X}$ & $\mathrm{X}$ & - & - \\
\hline (Kazi et al., 2012) & - & - & - & $\mathrm{X}$ \\
\hline This paper & - & $\mathrm{X}$ & $\mathrm{X}$ & $\mathrm{X}$ \\
\hline
\end{tabular}




\section{Linked Data Mashup Construction}

In this section, we describe the process of construction and publication of Linked Data Mashup (LDM). The integration process was based on the Linked Data Integration Framework (LDIF). LDIF suggests the following execution flow: $i$ ) Extraction of data sources; ii) Transformation of data (Triplification) and construction of exported of views; iii) Resolution of the identity through links owl:sameAs; iv) Data quality assessment and fusion and $v$ ) Data output (Schultz et al., 2012).

\subsection{Selected Sources}

In this work, the following criteria that were used for the selection of datasets: The data should have information about drugs description, commercial drugs, drugs risks, drug's indications and finally, the data must have relevance for non-specialist users. Also, preference is given to Brazilian or Portuguese data, especially for drugs sold only in Brazil and its risks.

Based on the previously listed criteria, four different datasets were selected, three of which are available from the Agência Brasileira de Vigilância Sanitária $(A N V I S A)^{1}$ or Brazilian Sanitary Surveillance Agency in English, and the last one belongs to the BIO2RDF project (Belleau et al., 2008). From ANVISA, we selected:

- Consumer Drug Prices (CDP)

- Government Drug Prices (GDP)

- Drug's risks in pregnant and breastfeeding (RPB)

CPD and GPD are found in the $X L S$ and $P D F$ file formats in website ${ }^{2}$, wherein this work the $X L S$ version was used. Both datasets have information about allopathic drugs, such as drug name, producer, barcode, therapeutic class, presentation, the active ingredient, and prices. The only difference between them is because the former has maximum selling prices for the average consumer, while the latter has maximum selling prices for government agencies.

The dataset RPB contains the risk categories of substances during the period of pregnancy and breastfeeding. This dataset can be found in webdocument ${ }^{3}$ and is only available in unstructured $P D F$.

The last dataset selected was the SIDER made available by the $B I O 2 R D F$ project, and can be found

\footnotetext{
${ }^{1} \mathrm{http}: / /$ portal.anvisa.gov.br/

${ }^{2} \mathrm{http}: / /$ portal.anvisa.gov.br/listas-de-precos

${ }^{3} \mathrm{http} / / /$ portal.anvisa.gov.br/documents/33880/2561889 /116.pdf/2292b730-2bd5-4acc-b378$10682 \mathrm{~b} 1 \mathrm{fc} 344$ ?version $=1.0$
}

in website ${ }^{4}$ already in the $R D F$ format. The dataset SIDER contains data about drugs, their indications, side effects, and different labels. However, the database only has data in English, not containing information about Brazilian drugs, making it necessary to translate it into Portuguese. This dataset has been selected because it contains information about the side effects of active principles, such information is needed to inform the risks of a drug.

\subsection{Vocabulary}

Because datasets have different structures, physical formats, and vocabularies, a mean was required to standardize access to information. The Linked Data approach uses ontologies to address such a problem. In the Linked Data paradigm, ontologies $O W L^{5}$ are used, which provide a representation of knowledge in a taxonomic way by through of a hierarchy of classes and properties, one of the objectives of $O W L$ is to structure data in a semantically understandable way by the machine allowing the inference of implicit information based on defined axioms.

The $O W L$ ontology provides a layer of semantic abstraction, allowing access to the integrated data in a transparent way to the user, in addition to using terms closer to their daily life, abstracting coded fields and giving definitions about terms, giving so the possibility of a greater understanding to the lay user.

In our work, a vocabulary was developed based on the data dictionaries of the original datasets, as well as other sources of knowledge about the domain such as sites, books, and manuals, having, in particular, the booklet "What we should know about drugs" available by ANVISA. In developing the vocabulary, it was always sought to conceptualize verbatim each term used, in addition to providing different alternative nomenclatures following the non-ontological sources cited before. The $O W L$ implementation can be found in ${ }^{7}$

\subsection{Exported views}

The exported view of a dataset consists of its representation using the vocabulary of the target ontology. The exported view represents an RDF view of the data

\footnotetext{
${ }^{4}$ http://download.bio2rdf.org/files/release/3/sider/sider.htm

${ }^{5}$ https://www.w3.org/OWL/

${ }^{6} \mathrm{http} / / / \mathrm{www} . v i g i l a n c i a s a n i t a r i a . s c . g o v \cdot b r / i n d e x . p h p /$ download/category/112-medicamentos?download=102: cartilhao-que-devemos-saber-sobre-medicamentos-anvisa

${ }^{7} \mathrm{https}: / /$ datahub.io/linkeddatamashupeducacional/data$\mathrm{med} / \mathrm{v} / 2$
} 
contained in the source, and this view can be materialized or virtual. In both views, there is a need for mapping rules to translate terms from the original dataset into the target vocabulary. The difference between materialized or virtual view lies in the fact that in the first case the data is physically converted to the RDF format and stored in a triplestore on which SPARQL queries will be made. While in the second the data is kept in its original format, where SPARQL queries will be made through a mediator that translates the SPARQL query into the native query language of the data (e.g., SQL) through the mappings. In this work we materialized the exported views

In this paper we will use the term triplification to refer to the process of generating $\mathrm{RDF}^{8}$ triples that represent the original data using the defined target vocabulary.

For the triplification of datasets $C D P$ and $G D P$, we imported the data into the relational database management system PostgreSQL ${ }^{9}$, due to the existence of matured tools in the conversion of relational databases for $R D F$. In this work, we used the tool $D 2 R Q$ (Bizer and Seaborne, 2004) that performs the transformation of relational bases to RDF by mapping the original schema to the desired target vocabulary. The mapping language used was $R 2 R M L^{10}$.

For the triplification of the $R P B$ dataset, manual conversion of the $P D F$ file to $C S V$ was required on account of the file's internal structure. But finally, the same process previously described was used for its triplification.

Finally, since the SIDER source already exists in the $R D F$ format, it was only necessary to use $S P A R Q L$ CONSTRUCT to mapping the original dataset to the desired vocabulary.

The result of this step was a set of four $R D F$ graphs with the target vocabulary representing each original dataset. However, it is still necessary to find out which resources represent the same object between the different $R D F$ graphs and merge them into one.

Figure 1 shows the exported views of the information contained in the original sources to existing concepts in the defined target ontology.

\subsection{Identity Resolution}

This step is responsible for discovering which different resources represent the same object in the real world to connect them via owl: sameAs links. These resources can be found on a single source or between

\footnotetext{
${ }^{8}$ https://www.w3.org/RDF/

${ }^{9} \mathrm{https}: / /$ www.postgresql.org/

${ }^{10} \mathrm{http}: / /$ www. w3. org/TR/r2rml/
}

the different sources, where the direct comparison between sources are only possible because there is already a guarantee that all sources have uniform structure and vocabulary because of the ontology.

In figure 2 is possible to see an example of identity resolution. In this example, two resources are representing the drug "Reopro", the CDP/Reopro (in left) representing it in the dataset CDP and GDP/Reopro (on the right) in the dataset GDP. Each of the resources has its properties, including its active principles, being these CDP/abciximab and GDP/abciximab. The active principle "abciximab" is also represented by different resources between the different datasets, and there is no connection between the resources. Without there being a connection between the resources of different datasets, there is no possibility, for example, to answer the risks of the drug "Reopro", since no dataset has such information individually.

The identity resolution will be responsible for discovering that the CDP/Reopro and GDP/Reopro resources represent the same object, thus constructing an owl:sameAs link between them. Furthermore, the links between the CDP/abciximab, GDP/abciximab, Sider/abciximab, and RPB/abciximab resources are also discovered at this stage. After creating the owl:sameAs links, it is already possible to merge information from several datasets about the drug "Reopro" so that it is possible to discover its risks.

For this step, the tool Silk Volz et al. (2009) was used. Silk uses user-specified rules to discover and generate links between resources. For this work, simple rules, such as strings treatment, comparison of values and averages, were used in general. For the most part, the defined rules have used the dc:title attribute that represents the title or nomenclature of the resource.

Links were generated between resources of the Drug, Laboratory, Therapeutic Class, Substance and Presentation classes. The other classes were not taken into account because their mappings themselves already guarantee the creation of resources with the same URI. Table 2 illustrates the number of links generated between sources.

\subsection{Quality Evaluation and Data Fusion}

After the identity resolution step, it is already possible to know through the owl: sameAs links which distinct resources represent the same real-world object. However, such resources are still represented by distinct URIs, so there is a need to merge such resources into a single one that will encompass all the properties and relationships of the originals. However, this 


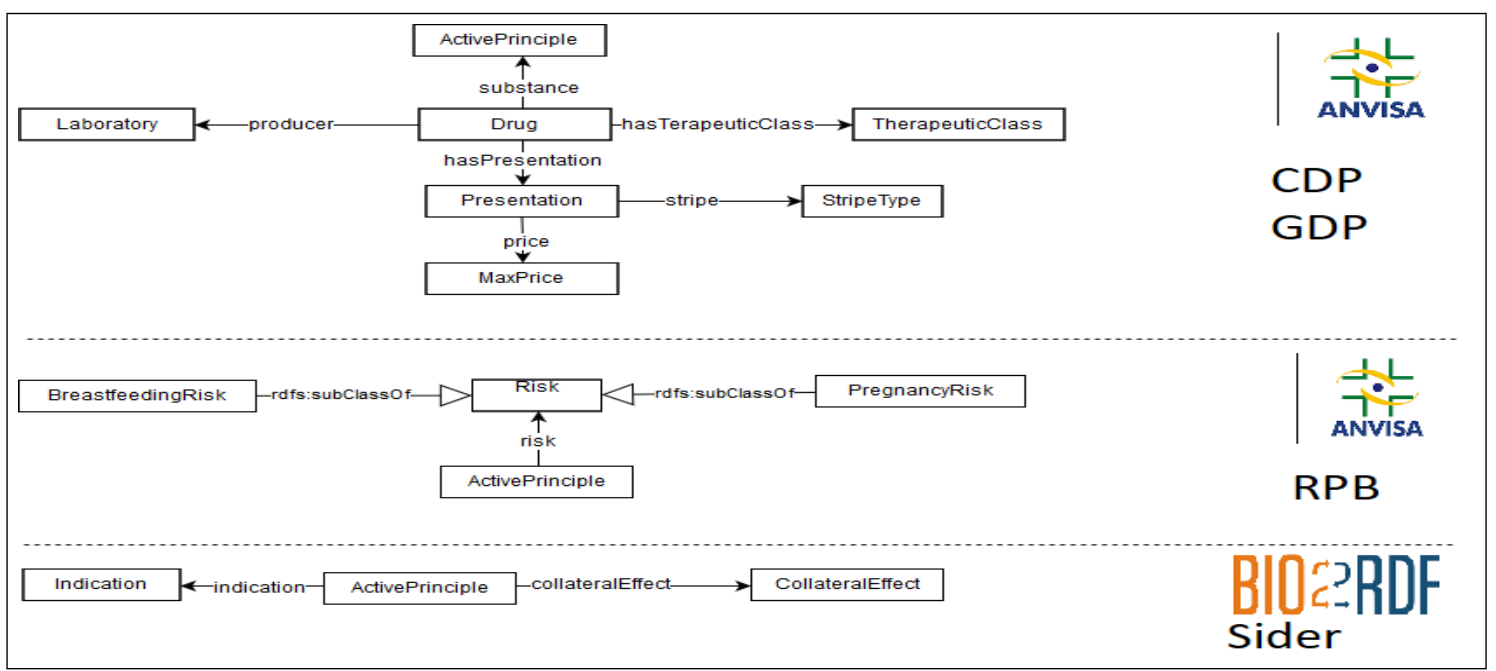

Figure 1: Datasets Exported Views.

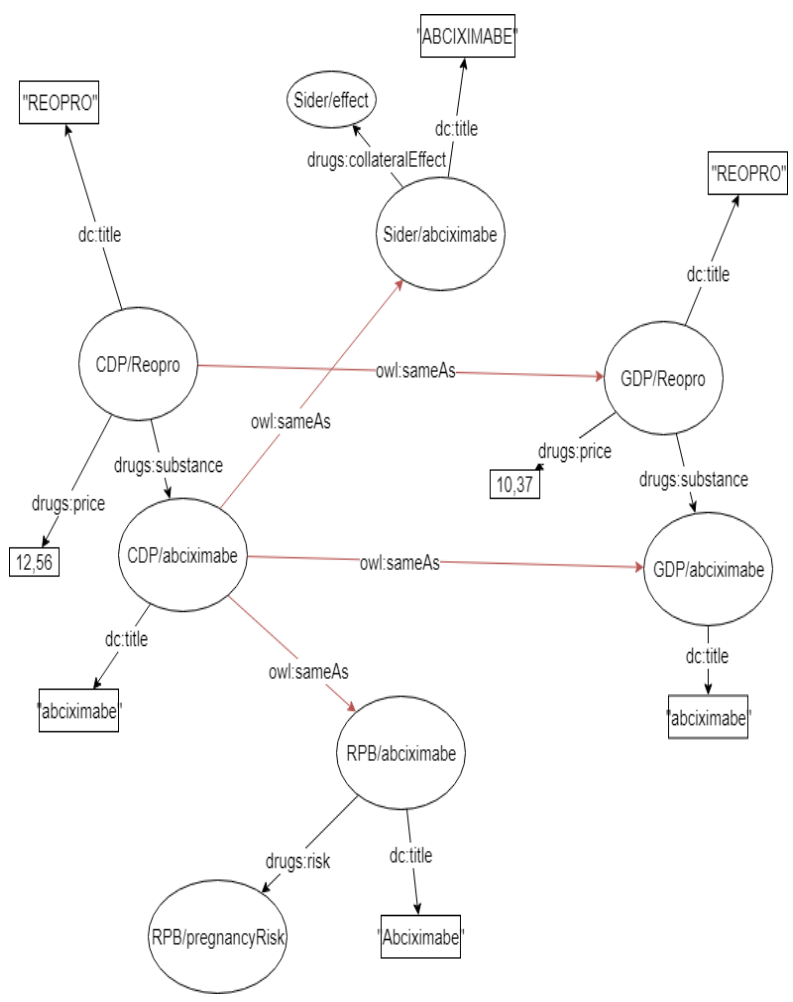

Figure 2: Example of Identity Resolution.

merge may cause some problems, such as repeated values for properties, conflicting between properties values, or incorrect values. So it's necessary to have the quality evaluation of the sources, this process defines the degree of priority of each source. After the quality evaluation, the data fusion and data cleaning process will be performed, choosing which information should be kept or deleted, based on the priority
Table 2: Generated sameAs Links by Class.

\begin{tabular}{|c|c|c|}
\hline Source A & Source B & Generated Links \\
\hline \multicolumn{3}{|c|}{ Drug } \\
\hline CDP & GDP & 8839 \\
\hline \multicolumn{3}{|c|}{ Laboratory } \\
\hline $\mathrm{CDP}$ & GDP & 25623 \\
\hline \multicolumn{3}{|c|}{ Therapeutic Class } \\
\hline $\mathrm{CDP}$ & GDP & 485 \\
\hline \multicolumn{3}{|c|}{ Presentation } \\
\hline $\mathrm{CDP}$ & GDP & 25623 \\
\hline \multicolumn{3}{|c|}{ Substance (Active Principle ) } \\
\hline CDP & GDP & 2164 \\
\hline CDP & Sider & 6401 \\
\hline CDP & RPB & 374 \\
\hline GDP & Sider & 6401 \\
\hline GDP & RPB & 323 \\
\hline RPB & Sider & 8956 \\
\hline Sider & Sider & 828175 \\
\hline
\end{tabular}

of each source.

In figure 3 , it is possible to observe the result of the fusion process of the drug "Reopro". After the fusion, the drug "Reopro" will be represented by a single resource, as well as its active principle. The resulting merged entities will contain all the information, previously scattered among the different datasets, where redundancy and inconsistency have already been solved.

For this step, we used the tool Sieve (Mendes et al., 2012). For the quality evaluation phase, the metric ScoredPrefixList was used, where for each source a weight was given in the following order: CDP, GDP, RPB and finally Sider. Such order was selected using manual analysis taking into account the quality of 


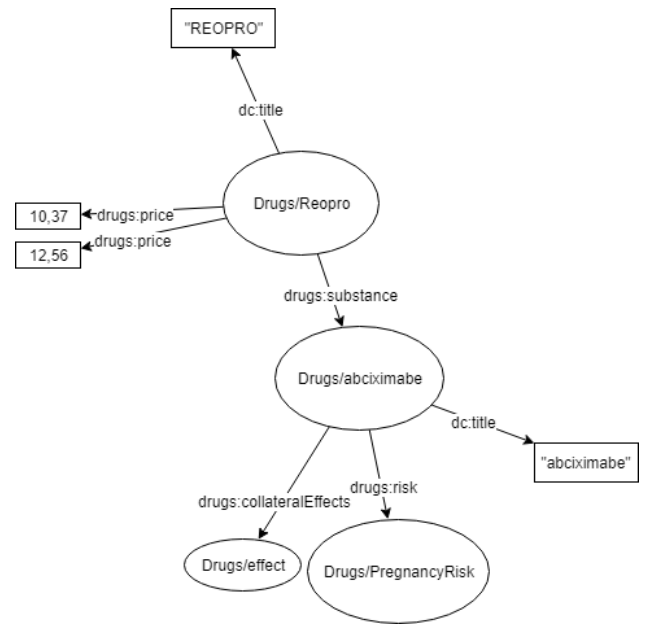

Figure 3: Example of resources fused.

how the data is structured, the scope of data, number of conflicts in original data and the number of links created between resources of the same source. This last rule comes from the intuition that if a source has many different resources that represent the same object, then the source was constructed less rigorously, so it will have a lower priority.

Fusion rules have been defined for cleaning only the classes Drug, Laboratory, Therapeutic Class, Substance, and Presentation, due possible fusion of such resources.

\subsection{Publication of the Linked Data Mashup}

At the end of the semantic integration process, we generated a dataset RDF containing the integrated view of the four original datasets, now following the same vocabulary and resources merged. This final dataset is called Linked Data Mashup.

The resulting dataset was then hosted in the virtuoso triplestore ${ }^{11}$, which provides a SPARQL endpoint capable of responding to $S P A R Q L$ queries via HTTP. This endpoint is accessed directly by the Medibot application. Moreover, the dump of the final dataset representing the LDM, in addition to the mapping files and the OWL implementation of the ontology can be accessed publicly via datahub ${ }^{12}$.

\footnotetext{
${ }^{11}$ https://virtuoso.openlinksw.com/

${ }^{12} \mathrm{https}: / /$ datahub.io/linkeddatamashupeducacional/data$\mathrm{med} / \mathrm{v} / 2$
}

\section{MediBot}

Although the ontology provides a layer of semantic abstraction with terms closer to the user, there are still problems in its access. To have access to the data, before it is necessary to know about the ontology's schema and knowledge about Semantic Web technologies, such as $R D F, O W L$ and SPARQL. A SPARQL query can be overly complicated, requiring technical expertise on the part of the user which would go against the purpose of this work which is to universalize knowledge about drugs for users of different profiles. Therefore, in this work, a data access interface was developed via natural language through a chatbot, called MediBot.

MediBot is a chatbot for the instant messenger Telegram, so that it can be used both via mobile application and via the web interface on the PC. MediBot was implemented in JavaScript using NodeJS. Currently, MediBot is able to answer questions in Portuguese. MediBot can is contacted via Telegram by id @ websemantica_bot.

In subsection 4.1 the MediBot architecture is presented. While subsection 4.2 addresses the workflow of the query process.

\subsection{MediBot's architecture}

Figure 4 shows MediBot's architecture. Its architecture is divided into three layers, user interface,server, and linked data mashup.

The first layer, the user interface layer is the interaction medium with the user, been represented by Telegram instant messenger. The user interface can be via mobile application in Android and IOS Telegram app or via web browser in Telegram website. Besides the mobile application and the website Telegram can also be accessed via a desktop application on PC. The reason for choosing the Telegram as a channel for the chatbot is because the tool already has a large user base and a robust infrastructure, besides having an easy API for the creation and use of chatbots.

The second layer is the server layer. This layer is responsible for the processing of requests and responses and is composed of two main components. The first component of this layer is the application server, which is responsible for receiving and processing the user's requests. The application server is directly connected to the Telegram access API; this component is the central module of the MediBot architecture. The application server receives the user request and builds the SPARQL query responsible for retrieving the desired information. Also, the application server is also responsible for sending the 


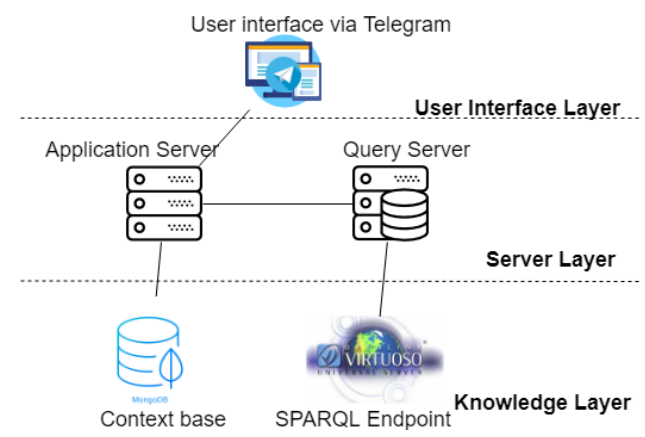

Figure 4: MediBot's architecture

SPARQL query to the query server and sending the response message to the user when it is ready.

The query server is responsible for receiving the SPARQL query and executing it on the SPARQL endpoint made available by the virtuoso. Besides, this component is also responsible for obtaining the response from the SPARQL query and constructing the response message to the user. The reason for splitting the server layer into two is because of the potentially long time to run the SPARQL query and generate the response message to the user. By dividing the application server into two components, it will not be locked during the processing of time-consuming queries, which would prevent it from receiving new requests and interactions that do not require queries.

The third and last layer is the Knowledge layer. This layer is responsible for storing the knowledge necessary for the chatbot to respond and interact with the user. This layer is composed of two components:

- Context Base: It is responsible for storing relevant context information during the interactive mode, such as personal information about the user such as name, surname and language, as well as information about the conversation flow as current interaction state, list of options presented, last message, term and object selected for consultation. Context information is stored in an instance of the non-relational object-oriented mongoDB ${ }^{13}$ database, which allows fast storage and quick retrieval of data stored in JSON, allowing direct processing by javascript code without the need for pre-processing the data.

- Linked Data Mashup: its description is detailed in chapter 3, loaded in the virtuoso triplestore that provides a SPARQL endpoint for the realization of queries.

\footnotetext{
${ }^{13}$ https://www.mongodb.com/
}

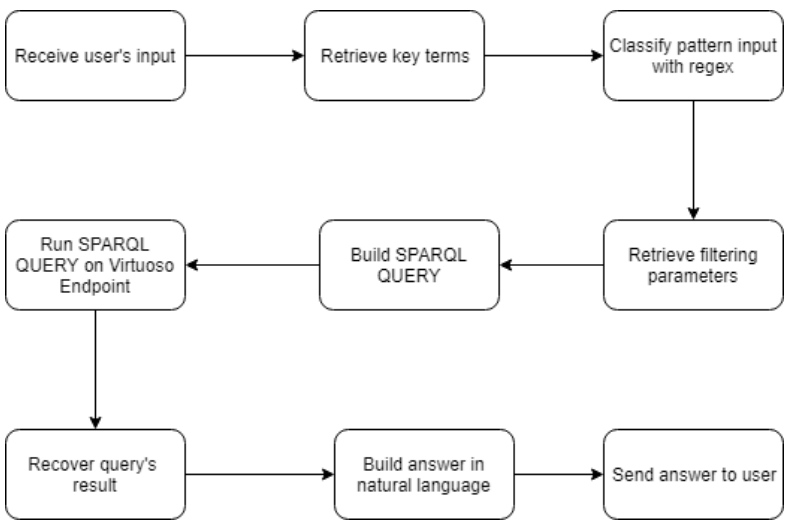

Figure 5: MediBot's workflow in quick response mode.

\subsection{Query workflow}

MediBot has two modes of operation, the first being the quick response mode and the second the interactive one. In quick response mode, MediBot has a set of SPARQL queries predefined and uses a simple regular expression evaluation approach to mapping the entry in one those predefined queries. During the input evaluation process, key terms and filtering parameters are retrieved. Key terms help classify into which type of query the entry should be mapped to while filtering parameters are used in FILTER clauses to restrict the query result to the specific intent of the user. Finally, the SPARQL query is built and performed via HTTP on the Virtuoso endpoint. Moreover, the building answer process also uses answers patters predefined. Figure 5 shows the workflow performed by MediBot.

Seven types of queries have been defined, which are shown in Table 3. While Figure 6 presents an image of MediBot responding to query 1 performed in natural language in Portuguese through the Telegram.

In addition to the quick response mode, MediBot has an interactive mode. While the former provides quick and easy access to information, the latter provides a versatile form of access to the knowledge contained in the sources. The main difference between the two modes lies in the interactive and conversational character of the interactive mode, while the interaction of the quick responses mode is summed up to individual questions and answers, the interactive mode performs information retrieval tasks in a conversational way where the context of the conversation and previous interactions influences the results of future interactions.

The interactive mode is oriented to finite tasks, where the user starts one task at a time through the sending of messages containing pre-established keywords; moreover, a task remains active as long as 
Table 3: Types of queries answered by MediBot in quick response mode.

\begin{tabular}{|l|l|}
\hline Type of query & Example \\
\hline $\begin{array}{l}\text { Drugs with } \\
\text { a principle active }\end{array}$ & $\begin{array}{l}\text { What are the drugs with } \\
\text { the substance dipyrone? }\end{array}$ \\
\hline $\begin{array}{l}\text { Definition } \\
\text { of terms in domain }\end{array}$ & Define therapeutic class \\
\hline $\begin{array}{l}\text { Informations about } \\
\text { certain drug }\end{array}$ & Talk about the drug aspirin \\
\hline $\begin{array}{l}\text { Indicate the risks } \\
\text { of a drug }\end{array}$ & $\begin{array}{l}\text { What are the risks of the } \\
\text { drug reopro? }\end{array}$ \\
\hline $\begin{array}{l}\text { List of drugs's } \\
\text { presentation }\end{array}$ & $\begin{array}{l}\text { What are the presentations } \\
\text { of the drug reopro? }\end{array}$ \\
\hline $\begin{array}{l}\text { Information about } \\
\text { barcode presentation }\end{array}$ & $\begin{array}{l}\text { Give information about bar } \\
\text { code presentation } \\
7896382701801\end{array}$ \\
\hline $\begin{array}{l}\text { Price of a presentation } \\
\text { with ICMS tax in } \\
\text { one State }\end{array}$ & $\begin{array}{l}\text { What are the price with } \\
\text { ICMS tax of presentation } \\
7896382701801\end{array}$ \\
\hline
\end{tabular}

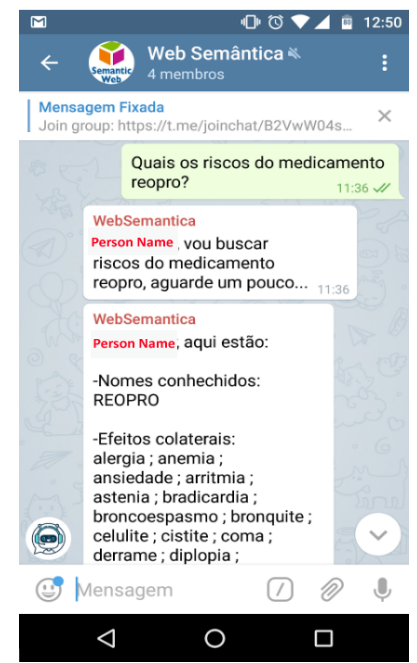

Figure 6: Example of Query in Portuguese on Telegram about the Drug's risks.

the user does not explicitly intend to finish it. However, during any point of an interactive mode task, the user can perform a quick response mode query, meanwhile, the already started task remains in stand by to be resumed any time the user wishes.

During interactive mode, two types of tasks can be performed, browser and query, which will be described later. However, the flow followed during the conversation is not free, following a well-defined pattern flow, where chatbot and user alternate their questions and answers. For decision of which next steps to be followed during a specific task's conversation, the MediBot uses information about past interactions during the same task (in this case called context), the current point of the task (in this case called state) and, in cases where the chatbot expects a response from the user, the message received message (in this case called input).

To enable continuous interaction between chatbot and user the interactive mode was implemented following a variation of the pushdown automaton approach, where the current state of the conversation or simply state is represented as a state of the automaton, the context of the conversation is represented as the auxiliary memory of the automaton, and the input of the user is represented by the input signal of the automaton. A remarkable aspect of the implementation of MediBot is the fact of the possibility of state change without an explicit input sequence, this is due to the fact that previous user responses may already have the information necessary for the state change without that there is a need for the user to reissue his intention. Due to simplicity and space constraints in this paper, the formal definition of the MediBot automaton will not be presented, however, in the figures 8 and 7 flowcharts are presented representing the tasks performed in the interactive mode, being possible to derive the automaton to from these flowcharts.

There are two types of possible tasks in interactive mode, which are:

- Browser Task: This task allows the user to navigate recursively on the existing terms in the knowledge base, including the ontology schema and its instances. When the user starts the task of browsing over a term, MediBot presents the different names, types and definitions of the term, besides presenting its properties and allow the user to select one of this to be the new pivot of the task, being able to navigate on the concepts of the sources. In figure 8 is presented the browser task's flow. In figure 10 is presented as an example of a browser task's interaction on Telegram.

- Query Task: This task allows the user to view data about instances contained in the knowledge base. Likewise the browser task, the query task is also recursively interactive. During this task, the user can ask to query the properties of a given instance. Upon receiving a query from a user, MediBot returns a list of instance's properties, giving the user the option to select one of these to view its values. If the selected property is a relation (owl:ObjectProperty) MediBot displays the list of instances as values for the property selected, which can be selected as the new pivot for the query task. If the selected property is a simple attribute (owl:DatatypeProperty), it simply displays a list with the constant values for the 


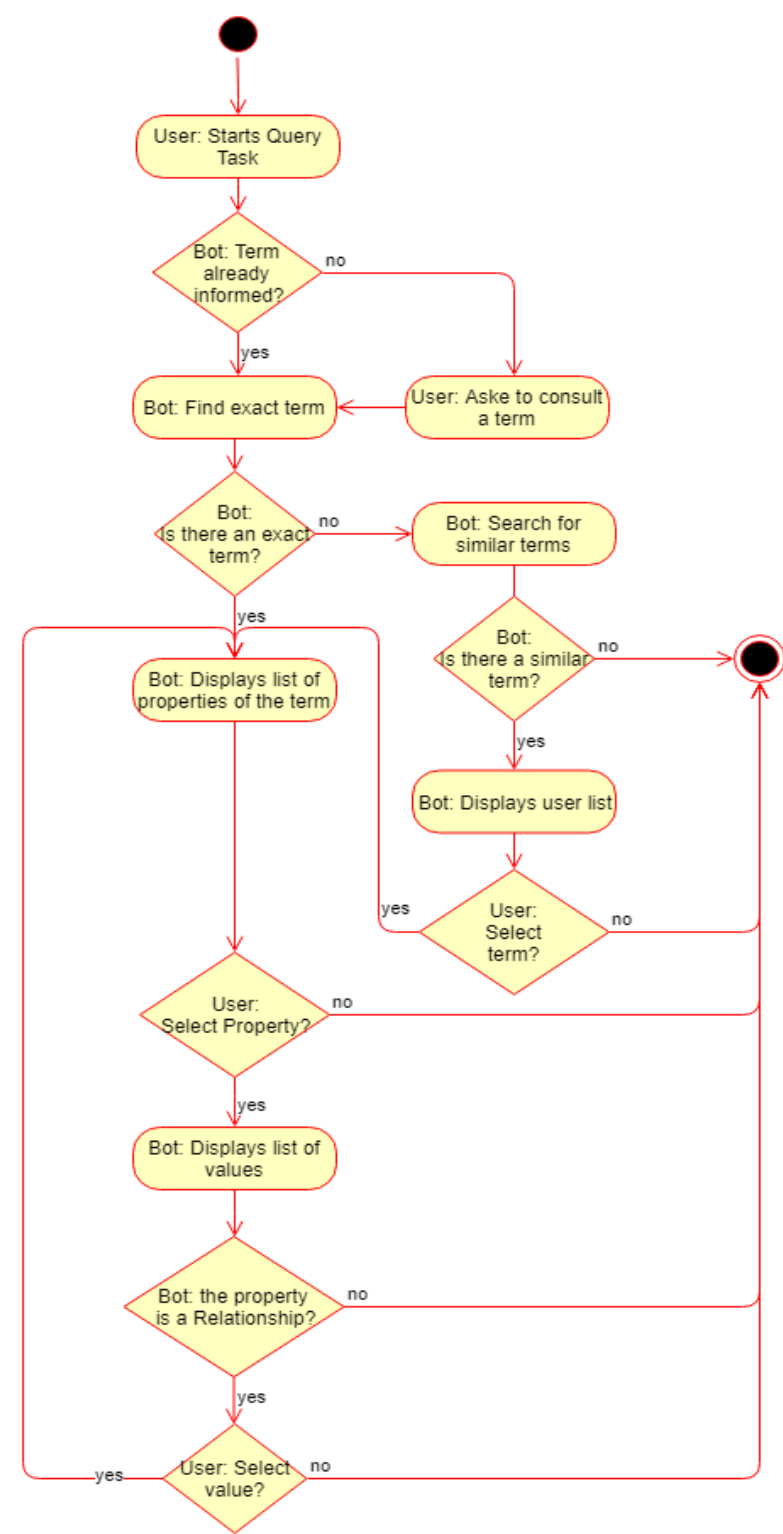

Figure 7: Query task's flowchart

property. In figure 7 is presented the query task's flow. In figure 9 is presented as an example of a query task's interaction on Telegram.

It is interesting to note that some steps of the query and browsers tasks are similar, such as terms list's display, these steps were implemented as a single state in the automaton, with the context being the only point of differentiation them. Another important implementation element lies in context's persistence since for being an online application and multi-user isn't feasible to keep the context in memory, so each time the context is changed or necessary it has resorted to the persistence mean MongoDB as described in subsec-

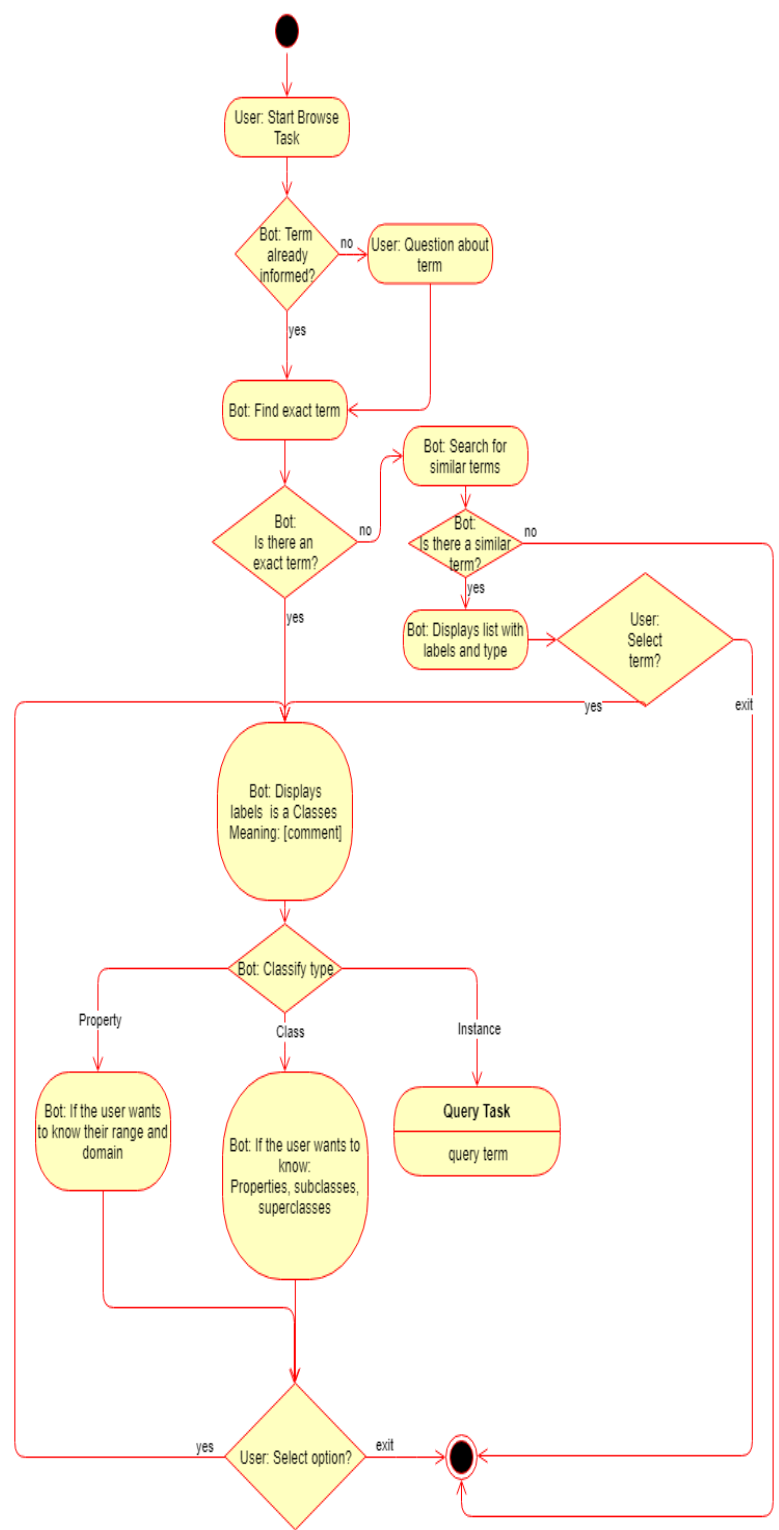

Figure 8: Browser task's flowchart

tion 4.1.

\section{Evaluation}

In this work, we select the task-based evaluation method described in (Konstantinova and Orasan, 2013) to measure the MediBot's usability degree. In this method are defined sets of tasks that are then requested to be performed by volunteers.

To evaluate the practical usefulness of MediBot, a set of 6 questions about information contained in the sources was elaborated. The questions were asked 


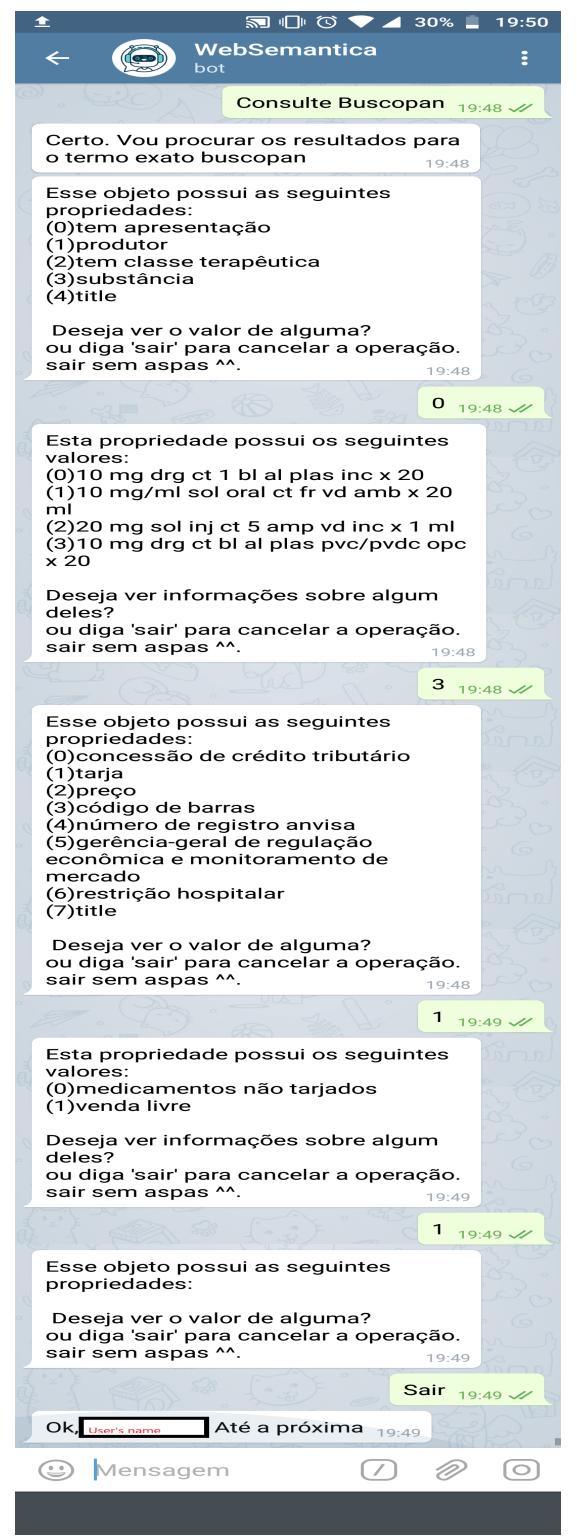

Figure 9: Example query task in telegram

for not involved with the project volunteer users who had to use the tool to respond to them. The questions can be divided into three levels of difficulty: (1) Easy, can be answered with a single interaction with the chatbot; (2) Medium, and can be answered with at least two interactions; and (3) Difficult, requiring more than two interactions with the chatbot.

The questions were asked through face-to-face interviews with volunteers. Ten volunteers, four men and six women participated in the study. The average age of the participants was 31 years, having a minimum age of 22 years and a maximum of 63 years. While none of them has technical knowledge about

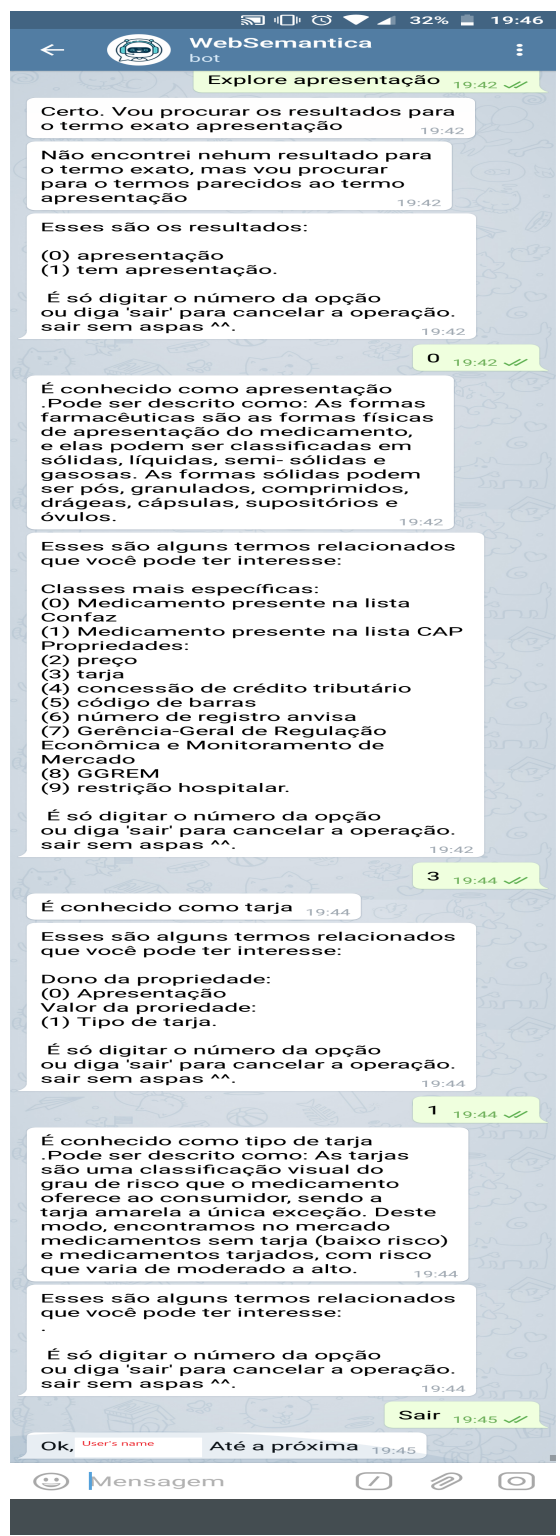

Figure 10: Example browser task in telegram

the field of medicines. Also, two of the participants had technical knowledge in information technology, among them one knowing about ontologies.

As evaluation criteria, It was using three aspects. The first criteria were the time needed to solve the questions, the second was the rate of correctness, and finally, the personal opinion of each evaluated that entered as a subjective criterion. In table 4 the result of the evaluation for each question and the final average is presented.

In the criterion of success rate dropouts were counted as an error, however, the time of such cases did not enter the meantime because it would cause 
Table 4: Evaluation result.

\begin{tabular}{|l|l|l|l|l|}
\hline ID_Question & Question & Mean Time(s) & Success Rate(\%) & Difficult \\
\hline Q001 & What is a black box remedy? & 30,53 & 100 & 1 \\
\hline Q002 & What are the risks of Tylenol? & 51,2 & 100 & 1 \\
\hline Q003 & $\begin{array}{l}\text { Which drugs have the same } \\
\text { active ingredient as Buscopan? }\end{array}$ & 142,10 & 80 & 2 \\
\hline Q004 & $\begin{array}{l}\text { What is Buscopan's maximum } \\
\text { price? }\end{array}$ & 358,96 & 20 & 2 \\
\hline Q005 & $\begin{array}{l}\text { What is the relationship between } \\
\text { a substance and a presentation? }\end{array}$ & 152,05 & 70 & 3 \\
\hline Q006 & $\begin{array}{l}\text { Which state has the lowest } \\
\text { maximum price for the } \\
\text { prescription drug orencia? }\end{array}$ & 320,34 & 10 & 3 \\
\hline Mean & & 175,86 & 63,33 & \\
\hline
\end{tabular}

distortions.

It is noteworthy that only queries Q001 and Q002 were able to be performed without prior knowledge about the ontology and the types of queries and their flows, while the others needed queries on such information. This fact was already expected, since MediBot has a limited set of pre-defined questions (which includes queries Q001 and Q002), whereas query and browser tasks require a correct starting point to be useful.

Another interesting point is that queries Q004 and Q006 took considerably longer, in addition to having a lower success rate. This fact can be explained because their answers are not represented in a factual way at the base. In the case of Q004, there was a need for a comparison operation, which in general users tried to compare all existing prices, which required many interactions, leading to a high dropout rate. There was the possibility to remove the number of possibilities taking in characteristics of the presentation, such as quantity, route of administration and others. Already in the case of query Q006, there was a need to have an understanding of how the state was related to price, where once again users attempted to make all comparisons. However, such a question could be resolved only by looking at the lowest value for taxes and which states adopted it.

During the reporting of opinions about the use of the tool the main points presented were that ontology's image and examples of queries were very useful. In addition to the preference for quick questions about query and browser tasks.

\section{Conclusion and future work}

The main objective of this work is to universalize access to information about drugs and their risks to users of different profiles, principally Brazilian and lay users in the domain.

To fulfill this goal, in this paper we presented MediBot, an ontology-based chatbot capable of responding to requests in natural language in Portuguese via the instant messenger Telegram. Medibot provides a data access interface without the need for the user to have prior knowledge about the structure of the ontology or technical knowledge about Semantic Web technologies.

Besides, in this work, we carried out the process of semantic data integration in the domain of drug's data, where the selection of data related to Brazil was preferred. Four separate datasets for integration were selected: "Consumer Drug Prices" (CDP), "Government Drug Prices" (GDP) and "Drug's risks in pregnant and breastfeeding" (RPB) made available by the National Agency Sanitary Surveillance (ANVISA in Portuguese), and Sider available from the BIO2RDF project.

Each of the datasets was originally in a different physical format, such as spreadsheets, $R D F$ or $P D F$. In addition, each original dataset had a different vocabulary. These facts show the justification of the use of technologies of Linked Data and Semantic Web to offer a layer of abstraction that allows transparent access to the data in an integrated way and with a vocabulary closer to that used by the user in their daily lives.

During the usability evaluating the process of MediBot, we realized that tasks that require comparison operations, such as Q004 and Q006, present a challenge for users because they require a great repetitive manual effort, so as future work we intend to implement automated ways to perform such operations, besides increasing the number of quick questions.

Still, as future work, we intend to develop an automatic and incremental data update mechanism to en- 
sure access to updated information efficiently. Moreover, we aim to integrate with a larger number of datasets, such as global dataset such as drug bank and registries from other countries, and use e-SUS datasets with microdata on the use of drugs.

\section{References}

Belleau, F., Nolin, M.-A., Tourigny, N., Rigault, P., and Morissette, J. (2008). Bio2rdf: towards a mashup to build bioinformatics knowledge systems. Journal of biomedical informatics, 41(5):706-716.

Bizer, C., Heath, T., and Berners-Lee, T. (2011). Linked data: The story so far. In Semantic services, interoperability and web applications: emerging concepts, pages 205-227. IGI Global.

Bizer, C. and Seaborne, A. (2004). D2rq-treating non-rdf databases as virtual rdf graphs. In Proceedings of the $3 r d$ international semantic web conference (ISWC2004), volume 2004. Proceedings of ISWC2004.

Corrêa, A. D., Caminha, J. d. R., Souza, C. A. M. d., and Alves, L. A. (2013). Uma abordagem sobre o uso de medicamentos nos livros didáticos de biologia como estratégia de promoção de saúde. Ciência \& Saúde Coletiva, 18:3071-3081.

Hoang, H. H., Cung, T. N.-P., Truong, D. K., Hwang, D., and Jung, J. J. (2014). Retracted: Semantic information integration with linked data mashups approaches. International Journal of Distributed Sensor Networks, 10(4):813875.

Jovanovik (2017). Consolidating drug data on a global scale using linked data. Journal of biomedical semantics, 8(1):3.

Kazi, H., Chowdhry, B., and Memon, Z. (2012). Medchatbot: An umls based chatbot for medical students. International Journal of Computer Applications, 55(17).

Konstantinova, N. and Orasan, C. (2013). Interactive question answering. In Emerging Applications of Natural Language Processing: Concepts and New Research, pages 149-169. IGI Global.

Mendes, P. N., Mühleisen, H., and Bizer, C. (2012). Sieve: linked data quality assessment and fusion. In Proceedings of the 2012 Joint EDBT/ICDT Workshops, pages 116-123. ACM.

Natsiavas, P., Koutkias, V., and Maglaveras, N. (2015). Exploring the capacity of open, linked data sources to assess adverse drug reaction signals. In $S W A T 4 L S$, pages 224-226.

Natsiavas, P., Maglaveras, N., and Koutkias, V. (2017). Evaluation of linked, open data sources for mining adverse drug reaction signals. In International Conference on Internet Science, pages 310-328. Springer.

Nováček, V., Vandenbussche, P.-Y., and Muñoz, E. (2017). Using drug similarities for discovery of possible adverse reactions. In AMIA Annual Symposium Proceedings. AMIA.
Pinto, M. C. X., Ferré, F., and Pinheiro, M. L. P. (2012). Potentially inappropriate medication use in a city of southeast brazil. Brazilian Journal of Pharmaceutical Sciences, 48(1):79-86.

Queneau, P., Bannwarth, B., Carpentier, F., Guliana, J.-M., Bouget, J., Trombert, B., Leverve, X., Lapostolle, F., Borron, S. W., and Adnet, F. (2007). Emergency department visits caused by adverse drug events. Drug Safety, 30(1):81-88.

Schultz, A., Matteini, A., Isele, R., Mendes, P. N., Bizer, C., and Becker, C. (2012). LDIF - A Framework for LargeScale Linked Data Integration. In 21 st WWW, Developers Track, page to appear.

Shadbolt, N., Berners-Lee, T., and Hall, W. (2006). The semantic web revisited. IEEE intelligent systems, 21(3):96-101.

SINITOX (2016). Sistema Nacional de Informações Toxicológicas registro de intoxicações no brasil. Accessed: 2018-09-17.

Sousa, H. W., Silva, J. L., and Neto, M. S. (2008). A importância do profissional farmacêutico no combate à automedicação no brasil. Revista eletrônica de farmácia, $5(1)$.

Vega-Gorgojo, G., Giese, M., Heggestøyl, S., Soylu, A., and Waaler, A. (2016). Pepesearch: Semantic data for the masses. PloS one, 11(3): $\mathrm{e} 0151573$.

Volz, J., Bizer, C., Gaedke, M., and Kobilarov, G. (2009). Silk-a link discovery framework for the web of data. LDOW, 538 . 